\title{
Predictors of gestational diabetes mellitus in a high- parity community in Saudi Arabia
}

M.A. Al-Rowaily' and M.A. Abolfotouh ${ }^{2}$

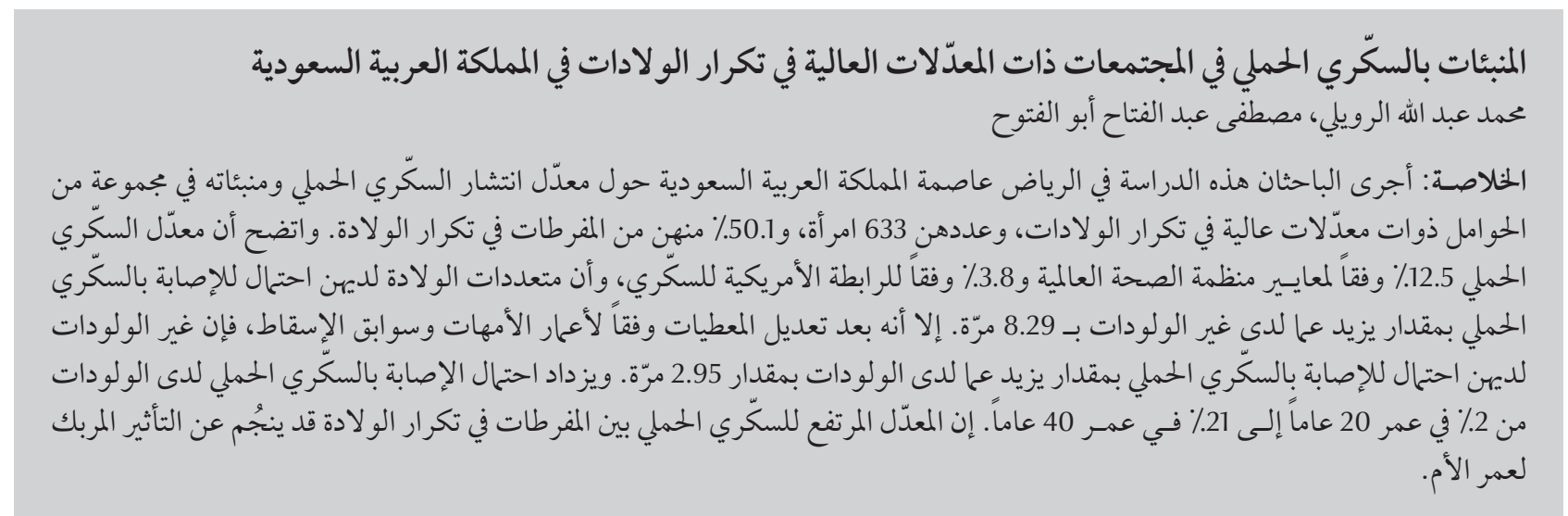

ABSTRACT A study in Riyadh, Saudi Arabia investigated the prevalence of gestational diabetes mellitus (GDM) and its predictors in a high-parity group of pregnant women ( $n=633,50.1 \%$ grand multiparas). The prevalence of GDM was $12.5 \%$ and $3.8 \%$ by World Health Organization and American Diabetes Association criteria respectively. Multiparous women were 8.29 times more likely to have GDM than nulliparous women. However, after adjustment for maternal age and history of abortion, nulliparous women were 2.95 times more likely to develop GDM than parous women. The probability of GDM for a parous woman increased from $2 \%$ to $21 \%$ when age increased from 20 to 40 years. The high rate of GDM among grand multiparas may be due to the confounding effect of maternal age.

Facteurs prédictifs du diabète gestationnel au sein d'une communauté à parité élevée en Arabie saoudite

RÉSUMÉ Une étude portant sur la prévalence du diabète gestationnel et sur ses facteurs prédictifs au sein d'un groupe de femmes enceintes à la parité élevée ( $n=633$, dont 50,1 \% de grandes multipares) a été réalisée à Riyad (Arabie saoudite). La prévalence de cette maladie était de $12,5 \%$ et de 3,8 \% selon les critères de l'OMS et de l'ADA, respectivement. La probabilité des femmes multipares de présenter un diabète gestationnel était 8,29 fois supérieure à celles des femmes nullipares. Toutefois, après ajustement des données en fonction de l'âge maternel et des antécédents d'avortement, les nullipares se sont révélées 2,95 fois plus susceptibles de présenter un diabète gestationnel que les femmes pares. La probabilité pour la femme pare de contracter cette maladie augmente de $2 \%$ à $21 \%$ entre 20 et 40 ans. Le fort taux de diabète gestationnel chez les grandes multipares peut être lié au facteur confusionnel de l'âge maternel.

${ }^{7}$ Department of Family Medicine; ${ }^{2}$ Biobanking Section, King Abdullah International Medical Research Centre, King Saud Bin-Abdulaziz University for Health Sciences, National Guard Health Affairs, Riyadh, Saudi Arabia (Correspondence to M.A. Abolfotouh: mabolfotouh@gmail.com).

Received: 23/04/08; accepted: 14/08/08 


\section{Introduction}

Gestational diabetes mellitus (GDM) is defined as carbohydrate intolerance of varying degrees of severity with onset or first recognition during pregnancy $[1,2]$. Women with GDM are at risk of pre-eclampsia and their babies are at risk of macrosomia and perinatal mortality $[3,4]$. The prevalence of GDM ranges from $1 \%$ to $14 \%$ of all pregnancies, depending on the population studied and the diagnostic tests and criteria employed [5]. The oral glucose tolerance test (OGTT) has for several decades been the international standard for the diagnosis of diabetes in nonpregnant adults. However, the criteria for defining diabetes differ between the American Diabetes Association (ADA) $[1,6]$ and the World Health Organization (WHO) [2], with the WHO now characterizing GDM as the joint category of diabetes and impaired glucose tolerance. The appropriateness of these different diagnostic criteria has been debated [7]; nevertheless women meeting the definition for GDM by either set of criteria are at greater risk of complications than women without the diagnosis.

Screening for GDM using riskfactor assessment is common practice internationally, although an obvious limitation is that data on risk factors related to prior obstetric events are not available for nulliparous women [8]. High parity (5+) is common in developing countries, especially in Arab nations such Saudi Arabia where large families are the norm [9]. The association between multiparity and pregnancy outcomes has been studied extensively [10-12], as has the relationship between parity and risk of type 2 diabetes [13]. However, the findings are inconsistent, and whether multiparity is related to adverse maternal and fetal outcomes remains uncertain.

The aim of the present study was to estimate the prevalence of GDMamong pregnant women attending the King Fahd National Guard hospital, Riyadh,
Saudi Arabia, using both WHO and ADA criteria and to investigate parity as a predictor of GDM in this high-parity community of pregnant women.

\section{Methods}

All pregnant women attending the antenatal clinic of King Fahd hospital, part of the National Guard Health Affairs services, are routinely subjected to an OGTT at 24-28 weeks gestation. The diagnosis of GDM is based upon the results of both the fasting sample and/ or the 2-hour OGTT test. The treating physician is notified immediately of any abnormal results, so that the woman can be referred to a specialized GDM clinic.

\section{Sample}

All pregnant women who attended the antenatal clinic during the period July 2005-July $2006(n=770)$, who had no previous history of diabetes without pregnancy were the target group of the present study. After excluding women who suffered an abortion before reaching 24-28 weeks gestation $(n=30)$ and those who refused the OGTT or did not attend for testing $(n=107)$, the final sample was 633 pregnant women.

\section{Data collection}

The OGTT was performed in the morning after a 12-hour overnight fast and 3 days of minimal carbohydrate diet and unlimited physical activity. Plasma glucose was determined before and 2 hours after administration of a $75 \mathrm{~g}$ glucose solution (Glucola) [5]. GDM was considered present if venous plasma glucose was equal or greater than the threshold values according to $\mathrm{WHO}$ criteria (fasting plasma glucose $\geq 7.0$ $\mathrm{mmol} / \mathrm{L}$ or plasma glucose 2 hours after glucose load $\geq 7.8 \mathrm{mmol} / \mathrm{L}$ ) [2] and according to the ADA criteria (fasting plasma glucose $\geq 5.3 \mathrm{mmol} / \mathrm{L}$ and plasma glucose 2 hours after glucose load $\geq 8.6 \mathrm{mmol} / \mathrm{L})[1]$.
A review was made of the records of all pregnant women to collect data on age, gravidity, parity and history of previous abortion. Parity was classified as: nulliparous (no previous viable pregnancy), multiparous (given birth to 1-4 children) and grand-multiparous (given birth to 5+ children) [10-12,14-16].

\section{Ethical issues}

The blood tests were performed free of charge as a part of the ongoing routine care of pregnant women at this centre and the women signed consent for management at the first booking antenatal visit. For the present study the records of all data were kept confidential. The study received ethical clearance from the institutional review board and the ethics committee of King Abdulaziz Medical City, National Guard Health Affairs in Riyadh.

\section{Analysis}

Data were analysed using SPSS, version 11. The chi-squared test was applied to compare categorical data. To investigate parity as a predictor of GDM, logistic regression analysis was applied with GDM as the dependant variable against maternal age, parity (nulliparous versus parous) and history of previous abortion (positive versus negative history) as independent variables. Analysis of covariance (ANCOVA) was used to compare means of fasting glucose and 2-h glucose, adjusted for age and history of abortion, between nulliparous and parous women. Significance was assumed if $P$-value was less than 0.05 .

\section{Results}

The 2-h OGTT was completed by, 633 (82\%) women. According to WHO criteria, GDM was diagnosed in 79 women, a prevalence of $12.5 \%$ (95\% CI: $10.0 \%-15.3 \%$ ), while according to ADA criteria there were only 24 women with GDM, a prevalence of 3.8\% (95\% CI: $2.4 \%-5.6 \%$ ) (Table 1). Only 24 women 


\begin{tabular}{|c|c|c|c|c|c|c|}
\hline \multirow[t]{3}{*}{ WHO criteria } & \multicolumn{4}{|c|}{ ADA criteria } & \multicolumn{2}{|c|}{ Total } \\
\hline & \multicolumn{2}{|c|}{ Diabetes } & \multicolumn{2}{|c|}{ No diabetes } & \multirow[b]{2}{*}{ No. } & \multirow[b]{2}{*}{$\%$} \\
\hline & No. & $\%$ & No. & $\%$ & & \\
\hline Diabetes & 24 & 29.6 & 55 & 70.4 & 79 & 12.5 \\
\hline No diabetes & 0 & - & 554 & 100.0 & 554 & 87.5 \\
\hline Total & 24 & 3.8 & 609 & 96.2 & 633 & 100.0 \\
\hline
\end{tabular}

$k=0.433, \mathrm{P}<0.001$.

Sources: WHO 1999 [2]; ADA 2000 [1].

(100\% of ADA-defined cases and 29.6\% of WHO-defined cases) were positive by both criteria. All ADA-defined cases were detected by WHO criteria, while only $29.6 \%$ of the WHO-defined cases were detected by ADA criteria.

Among this group, 31 (50.1\%) were grand multiparas. Table 2 shows the univariate association of GDM with maternal age, parity and history of abortion. The prevalence of GDM according to WHO criteria was significantly associated with maternal age $\left(\chi_{\mathrm{LT}}^{2}=2.89\right.$, $P<0.001)$ and parity $\left(\chi^{2}=18.06, P<\right.$ $0.001)$ but not with a history of abortion $\left(\chi^{2}=4.85, \mathrm{df}=3, P=0.18\right)$. There was a significant and progressive increased prevalence of GDM with increasing maternal age, from $5.6 \%$ at 20-29 years to $28.3 \%$ among women aged $40+$ years. The prevalence of GDM was 9.5\% (95\% CI:3.6\%-19.6\%) among the nulliparous women, $6.3 \%$ (95\% CI: 3.9\%-10.5\%) among the multiparas (1-4 live births) and $18.2 \%$ (95\% CI: $14.1 \%-23.2 \%$ ) among grand-multiparous women (5+ live births). Multiparous women were 8 times more likely to have GDM [unadjusted odds ratio (OR) 8.29, 95\% CI: 1.01-179.87] than nulliparous women.
Logistic regression analysis was applied, with maternal age, parity and history of abortion as independent variables against GDM as the dependant variable (Table 3). When the regression model was assessed for goodness of fit, $87.1 \%$ of all cases were found to be correctly classified according to the presence of GDM. Table 3 shows the estimated coefficients and their exponential from the logistic regression model that predicted GDM from a constant and the variables: age in years, parity (1 for nulliparous) and history of abortion (1 for positive history). The $\chi^{2}$-value for the model was 30.55

Table 2 Prevalence of gestational diabetes mellitus (GDM) among pregnant women by World Health Organization criteria according to selected maternal characteristics

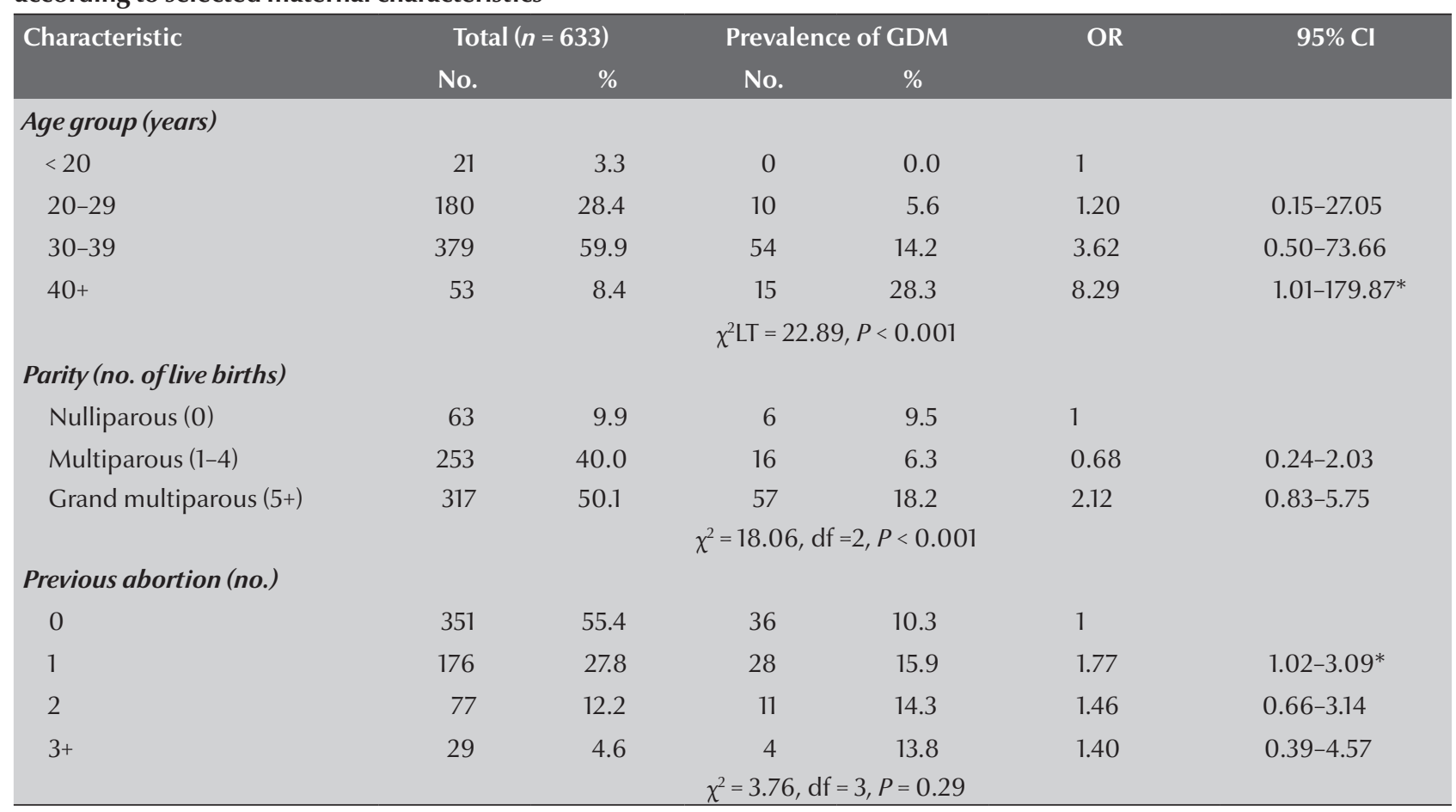

${ }^{*} \mathrm{P}<0.05$.

$O R=$ odds ratio; $\mathrm{Cl}=$ confidence interval.. 


\begin{tabular}{lccccc}
\hline \multicolumn{5}{l}{ Table 3 Logistic regression analysis of gestational diabetes mellitus with maternal age, parity and history of abortion } \\
\hline Independent variable & $\boldsymbol{\beta}$ & $\mathrm{SE}$ & $P$-value & $\mathrm{OR}$ & $\mathbf{9 5 \%} \mathrm{Cl}$ \\
Maternal age & 0.0124 & 0.025 & $<0.001$ & 1.13 & $1.08-1.19$ \\
Abortion (+ve history = 1) & 0.341 & 0.253 & 0.18 & 1.41 & $0.86-2.31$ \\
Parity (nulliparous = 1) & 1.083 & 0.540 & 0.045 & 2.95 & $1.03-8.51$ \\
Constant & -6.288 & 0.905 & $<0.001$ & 0.002 & \\
\hline
\end{tabular}

$S E=$ standard error; $O R=$ odds ratio; $C I=$ confidence interval.

$(P<0.0001)$. Age was the most highly significant predictor of GDM, with increasing maternal age associated with a greater likelihood of GDM (OR 1.13; $95 \%$ CI: $1.08-1.19, P<0.001]$. Nulliparity was significantly associated with the occurrence of GDM after adjusting for maternal age and history of abortion (OR 2.95; 95\% CI: 1.03-8.51, $P<0.05$ ). A history of abortion was not associated with GDM after this adjustment (OR 1.41; 95\% CI: $0.86-2.31, P=0.18$ ).

Given the previous coefficients, the logistic regression equation for the probability of occurrence of GDM was: $Z=-6.288+0.124$ (age in years) +0.341 (history of abortion) + 1.038 (parity). Applying this to a pregnant woman who is 20 years with values of 0 for the remaining independent variables (i.e. a parous woman with no history of abortion), we find: $Z=-6.288+0.124$ $(20)=-3.808$. The probability of GDM is then estimated as: $1 / 1+\mathrm{e}-\mathrm{z}=1 / 1$ $+\mathrm{e}-(-3.808)=0.02$. Based on this, we would predict that a nulliparous woman is only $2 \%$ likely to get GDM. Following the same procedure for a woman aged 40 years, the estimated probability of GDM was: $1 / 1+\mathrm{e}-(-1.328)=0.21$, i.e. GDM is $21 \%$ likely to occur at this age. However, if this same woman of 40 years of age was nulliparous, then applying the value of 1 for parity, the estimated probability of GDM is $1 / 1$ $+\mathrm{e}-(-0.245)=0.44$, i.e. the probability will rise to $44 \%$, indicating a higher possibility of GDM.

Table 4 compares nulliparous and parous women according to selected variables. In the bivariate analysis, parous women had a significantly higher mean maternal age [32.62 (SD 5.47) years versus $22.40(\mathrm{SD} 4.01)$ years] $(t=19.83, P<0.001)$ and higher rate of positive history of previous abortion $(48.4 \%$ versus $12.5 \%)\left(\chi^{2}=34.02\right.$, $P<0.001)$. The groups were similar in terms of the results of the OGTT. However, after adjustment for age and previous history of abortion, nulliparous women had significantly higher mean 2-h glucose values $(P<0.01)$ and higher prevalence of $\operatorname{GDM}(P=0.045)$.

\section{Discussion}

GDM is an asymptomatic condition most of the time and the effectiveness of its detection has not been adequately tested. Based on ADA criteria for diagnosis of GDM, $3.8 \%$ of the studied pregnant women who completed the $2-h$ $75 \mathrm{~g}$ OGTT had a diagnosis of GDM. The corresponding figure according to $\mathrm{WHO}$ criteria for GDM was $12.5 \%$. The latter figure is comparable with the incidence in Jeddah city (12.5\%) [17] but is higher than the figures reported for Saudis living in the Dammam [18] or Riyadh [19] areas. It is compatible with figures from other nearby countries [20], but still higher than those of some developed countries [21]. However, comparison of the incidence in different communities may lack validity due to the diversity in the diagnostic criteria used.

The findings of the present study revealed that all cases of GDM by ADA criteria were detected by WHO criteria, while less than $30 \%$ of cases detected by WHO criteria were also diagnosed by ADA criteria. Meanwhile, the number of GDM cases according to WHO criteria $(n=79)$ was more than 3 times that for cases detected by ADA ( $n=$ 24). This is consistent with the guidelines of the Society of Obstetricians and

\begin{tabular}{lcccc}
\hline \multicolumn{1}{l}{ Table 4 Comparison between nulliparous and parous women according to selected variables } \\
\hline Variable & $\begin{array}{c}\text { Nulliparous } \\
(\boldsymbol{n}=\mathbf{6 3 )}\end{array}$ & $\begin{array}{c}\text { Parous } \\
(\boldsymbol{n}=\mathbf{5 7 2})\end{array}$ & $\begin{array}{c}\boldsymbol{P} \text {-value } \\
\text { (unadjusted) }\end{array}$ & $\begin{array}{c}\boldsymbol{P} \text {-value } \\
(\mathbf{a d j u s t e d})\end{array}$ \\
Mean (SD) maternal age (years) & $22.4(4.0)$ & $32.6(5.5)$ & $t=19.83, P<0.001$ & - \\
Positive history of abortion (\%) & 12.5 & 48.4 & $\chi^{2}=34.02, P<0.001$ & - \\
Fasting plasma glucose (mmol/L) & 4.92 & 5.02 & 0.24 & 0.61 \\
2-hour plasma glucose (mmol/L) & 6.08 & 6.15 & 0.71 & 0.01 \\
Prevalence of GDM (\%) & 9.5 & 12.8 & 0.30 & 0.045 \\
\hline
\end{tabular}

Data are shown for women whose data were available.

aAdjustment was made for maternal age and history of abortion.

$G D M=$ gestational diabetes mellitus; $S D=$ standard deviation . 
Gynaecologists of Canada, who reported that the use of WHO criteria would approximately double the number of women diagnosed with GDM [22].

According to Schmidt et al. [4], women meeting the definition for GDM by either WHO or ADA criteria were at greater risk of pre-eclampsia and their babies were at greater risk of macrosomia and perinatal mortality than women without GDM. Thus, until consensus criteria are reached, these 2 criteria are valid options for the detection of adverse pregnancy outcomes. However, assuming that effective treatment is available, WHO criteria, by identifying a larger number of cases, may have greater potential for screening.

High parity (parity $5+$ ) is common in developing countries, especially in Arab nations where large families are the norm. The incidence of grand multiparity ranges from $0.6 \%$ in Croatia and Hong Kong [15] to 5\% in Trinidad [14], $11 \%$ in Nigeria [23] and above $30 \%$ in the United Arab Emirates [24] and Sudan [11]. In the present study, $50.1 \%$ of all pregnant women were grand multiparas. Many studies have been conducted to investigate the relationship between high parity and adverse birth outcome [10-12,14-16]. The relationship between parity and risk of type 2 diabetes has also been examined [13]. Most reported associations between parity and diabetes have not been adjusted for age or body adiposity, both of which are likely to be important confounding factors $[13,25]$. In studies that have presented results adjusted for age and adiposity $[13,26,27]$, the findings have been highly inconsistent. In the present study, when parity was used as an ordinal variable, GDM was significantly more prevalent among nulliparas as well as grand-multiparas, while it showed the lowest prevalence among multiparous women (1-4 live births). However, when using this parity variable as a dichotomous variable (nulliparous versus parous), no significant difference was detected in GDM prevalence between the 2 groups in the bivariate analysis. After adjustment by logistic regression analysis for both maternal age and history of previous abortion (both showed highly significant differences between the 2 groups), a significant association between parity and GDM was detected, so that GDM was about 3 times more likely to occur in nulliparous than in parous women. Meanwhile, the mean value of the 2-h OGTT, after adjusting for age and history of abortion, using ANCOVA, was significantly higher among the nulliparous group than among the parous group $(P=0.01)$.

It has been reported that many complications are associated with grand multiparity, including GDM, which was shown to increase with maternal age [16]. That is to say, the association between parity and adverse maternal complications might actually be due to the confounding effect of maternal age. In the present study, maternal age was strongly associated with the occurrence of GDM, with pregnant women aged $40+$ years about 8 times more likely to have gestational diabetes $(\mathrm{OR}=8.29)$ compared with younger women. Even after adjusting for parity and history of previous abortion using logistic regression analysis, maternal age was still a significant independent predictor of GDM. The results of the present study revealed that the probability of occurrence of GDM increased from $2 \%$ to $21 \%$ when age increased from 20 to 40 years, and this later probability was doubled (i.e. 44\%) for women who were nulliparous.

Some limitations have to be addressed in the present study. First it was a relatively small sample size that might not allow the results to be generalized. Secondly we used only 2 of the 3 readings of plasma glucose recommended for diagnosing GDM by the ADA (fasting and 2-h, but not 1-h readings).

From the findings of this study, and considering its limitations, we conclude that:

- According toWHO criteria the prevalence of GDM at King Fahd National Guard Hospital is high (12.5\%).

- Nulliparity could be considered as a significant independent predictor of GDM until further studies with higher numbers of nulliparas are done to support this finding.

- Maternal age is a significant predictor of GDM, and the high rate of GDM among grand multiparas could be due to the confounding effect of maternal age.

\section{Acknowledgements}

This project was approved by the institutional review board of King Abdulaziz Medical City, National Guard Health Affairs, Riyadh, Saudi Arabia. The authors would like to express their gratitude and thanks to the staff members of King Abdullah International Medical Research Center for their constructive support throughout the study.

\section{References}

1. American Diabetes Association. Gestational diabetes mellitus. Diabetes care, 2000, 23(Suppl. 1):S77-9.

2. Definition, diagnosis and classification of diabetes mellitus and its complications: Report of a WHO Consultation. Part 1: diagnosis and classification of diabetes mellitus. Geneva, World Health Organization, 1999 (WHO/NCD/NCS/99.2).

3. Kjos SL, Buchanan TA. Gestational diabetes. New England journal of medicine, 1999, 341:1749-56. 
4. Schmidt Ml et al. Gestational diabetes mellitus diagnosed with a 2-h 75-g oral glucose tolerance test and adverse pregnancy outcomes. Diabetes care, 2001, 24:1151-5.

5. Diabetes mellitus. Report of a WHO study group. Geneva, World Health Organization, 1985 (WHO Technical Report Series, No. 727).

6. Metzger BE, Coustan DR. Summary and recommendations of the Fourth International Workshop-Conference on Gestational Diabetes Mellitus. The Organizing Committee. Diabetes care, 1998, 21(Suppl. 1):B161-7.

7. Gabir MM et al. The 1997 American Diabetes Association and 1999 World Health Organization criteria for hyperglycemia in the diagnosis and prediction of diabetes. Diabetes care, 2000, 23(8):1108-12.

8. Russell MA, Carpenter MW, Coustan DR. Screening and diagnosis of gestational diabetes mellitus. Clinical obstetrics and gynecology, 2007, 50(4):949-58.

9. Kumari AS, Badrinath P. Extreme grandmultiparity: is it an obstetric risk factor? European journal of obstetrics, gynecology, and reproductive biology, 2002, 101:22-5.

10. Bai J et al. Parity and pregnancy outcomes. American journal of obstetrics and gynecology, 2002, 186:274-8.

11. Aziz FA. Pregnancy and labor of grand multiparous Sudanese women. International journal of gynecology and obstetrics, 1980, 18:144-6.

12. Eidelman Al et al. The grand multipara: is she still at risk? American journal of obstetrics and gynecology, 1988, 158:389-92.

13. Boyko EJ et al. Effects of childbearing on glucose tolerance and NIDDM prevalence. Diabetes care, 1990, 13:848-54.

14. Roopnarinesingh S, Ramsewak S, Reddy S. Complications of grand multiparity. West Indian medical journal, 1988, 37:222-5.

15. King PA, Duthie SJ, Ma HK. Grand multiparity: a reappraisal of the risks. International journal of gynecology and obstetrics, 1991, 36:13-6.

16. Aliyu $\mathrm{MH}$ et al. High parity and adverse birth outcomes: exploring the maze. Birth, 2005, 32:1.
17. Ardawi MSM et al. Screening for gestational diabetes mellitus in pregnant females. Saudi medical journal, 2000, 21(2):155-60.

18. Khwaja SS, Al-Suleiman SA, Al-Sibai MH. Screening for gestational diabetes in a teaching hospital in Saudi Arabia. Australian and New Zealand journal of obstetrics \& gynaecology, 1989, 29:209-11.

19. Al-Shawaf T, Akiel A, Moghbraby SA. Gestational diabetes and impaired glucose tolerance of pregnancy in Riyadh. British journal of obstetrics and gynaecology, 1988, 95:84-90.

20. Al-Mahroos S et al. A population-based screening for gestational diabetes mellitus in non-diabetic women in Bahrain. Annals of Saudi medicine, 2005, 25:29-33.

21. Naylor CD et al. Cesarean delivery in relation to birth weight and gestational glucose tolerance: pathophysiology or practice style? Toronto Trihospital Gestational Diabetes Investigators. Journal of the American Medical Association, 1996, 275:1165-70.

22. Society of Obstetricians and Gynaecologists of Canada. Screening for gestational diabetes. Journal of obstetrics and gynaecology Canada, 2002, 24(11):894-903.

23. Ozumba BC, Igwegbe AO. The challenge of grandmultiparity in Nigerian obstetric practice. International journal of gynecology and obstetrics, 1992, 37:259-64.

24. Rizk DE, Khalfan M, Ezimokhai M. Obstetric outcome in grand multipara in the United Arab Emirates. A case control study. Archives of gynecology and obstetrics, 2001, 264:194-8.

25. Manson JE et al. Parity and incidence of non-insulin dependent diabetes mellitus. American journal of medicine, 1992, 93:13-8,

26. Cowan LD et al. Parity, postmenopausal estrogen use, and cardiovascular disease risk factors among American Indian women: the Strong Heart Study. Journal of women's health, 1997, 4:441-9.

27. Kritz-Silverstein D et al. Relation of pregnancy history to insulin levels in older, nondiabetic women. American journal of epidemiology, 1994, 140:375-82. 